\title{
Does Smoking and Alcohol Increase the Risk of COVID-19? A Review
}

\author{
Ankit Mahajan ${ }^{1}$ Ujwal Singh ${ }^{2}$ Pankaj Kumar ${ }^{3}$ \\ ${ }^{1}$ Department of Periodontology and Implantology, H. P. Government \\ Dental College and Hospital Shimla, Shimla, Himachal Pradesh, India \\ 2 Department of Periodontology and Implantology, Private Dental \\ Practitioner, New Delhi, India \\ ${ }^{3}$ Department of Dentistry, Tata Central Hospital, Jharia, Jharkhand, \\ India
}

Dent J Adv Stud 2022;10:51-54.

\begin{abstract}
Address for correspondence Ankit Mahajan, MDS, Department of Periodontology and Implantology, H. P. Government Dental College and Hospital, Shimla 171001, Himachal Pradesh, India (e-mail: umasharma957@yahoo.com).
\end{abstract}

\begin{abstract}
Keywords

- COVID-19

- SARS-CoV-2

- smoking

- alcohol

- risk

The first case of pneumonia of unknown origin was identified in Wuhan, the capital city of Hubei Province situated in the Republic of China. The pathogenic organism that has been identified as a causative organism is a novel enveloped RNA betacoronavirus, which has been designated as severe acute respiratory syndrome coronavirus-2 (SARSCoV-2). This virus has been found to have a similar phylogeny to SARS-CoV. The novel coronavirus or COVID-19 can be symptomized through clinical manifestations like pyrexia or fever, cough, dyspnea/difficulty in breathing, myalgia/muscle pain, and constant fatigue. COVID-19 can be transmitted through respiratory tract secretions. It mainly results in respiratory tract infections and the development of severe pneumonia in infected patients. Severe disease may ultimately cause death due to progressive respiratory failure. The coronavirus disease 2019 or COVID-19 has been declared a public health emergency of international proportions by the World Health Organization (WHO). Thus, an analysis of cases might help in the identification of the disease's defining clinical characteristics, association with underlying lifestyle habits, and disease severity. This present article studied the probability of smoking and alcohol consumption in increasing the risk involved in increasing the severity of COVID-19 infection among affected subjects.
\end{abstract}

\section{Introduction}

The coronaviruses were first discovered in the 1960s. These viruses were classified under the Coronaviridae family. This family of virus forms the largest among the "Nidovirales" order. This family is composed of two subfamilies: Orthocoronavirinae and Torovirinae. Orthocoronavirinae includes a total of four genera- $\alpha-, \beta-$, gamma-, and delta-Coronavi- ruses. The $\alpha$ - and the $\beta$-coronaviruses are found among mammals which include bats. The gamma variety infects all avian and few mammalian species, while the delta variety infects both the avian and mammalian species. ${ }^{1}$

The designation severe acute respiratory syndrome Coronavirus-2 (SARS-CoV-2) was proposed by the "Coronaviridae Study Group of the International Committee on Taxonomy of Viruses," according to its taxonomy and phylogeny. Till published online November 16, 2021
DOI https://doi.org/ 10.1055/s-0041-1736490. ISSN 2321-1482. (c) 2021. Bhojia Dental College and Hospital affiliated to Himachal Pradesh University. All rights reserved.

This is an open access article published by Thieme under the terms of the Creative Commons Attribution-NonDerivative-NonCommercial-License, permitting copying and reproduction so long as the original work is given appropriate credit. Contents may not be used for commercial purposes, or adapted, remixed, transformed or built upon. (https://creativecommons.org/ licenses/by-nc-nd/4.0/)

Thieme Medical and Scientific Publishers Pvt. Ltd., A-12, 2nd Floor, Sector 2, Noida-201301 UP, India 
April 4, 2020, there were overall 1,117,942 confirmed cases of COVID-19 cases with 59,201 fatalities across the world. ${ }^{2}$

The novel coronavirus (also known as, 2019-nCoV and SARS-CoV-2) was first described in December 2019 in pneumonia-affected subjects belonging to Wuhan, which is situated in China. The rapidly rising numbers of cases of this virus around the world have led to a generalized fear, which has been termed as "corophobia." This virus is transmitted through the respiratory tract route. ${ }^{3}$ COVID-19 can manifest clinically as asymptomatic carrier, acute respiratory symptoms, and pneumonia. Neonates, children, and older adults can also get infected alongside. ${ }^{4}$ The most prevalent preexisting diseases found in COVID-19 patients under hospitalization include hypertension, cardiovascular disease, habit of smoking, chronic renal diseases, malignancies, and chronic obstructive pulmonary disease (COPD). This is the third most common contagious disease following Middle Eastern respiratory syndrome (MERS) and SARS. The most challenging scenarios which make tackling of this virus include its diagnosis, exact mode of transmission, the longer incubation period (lasting from 3-14 days) and insufficient numbers of available protective resources. The exact rate of SARS-CoV-2 is unknown, although human-to-human transmission is evident due to its wide prevalence among family members and workers related to health care services. Since it is a newly identified pathogenic microorganism, there is no acquired immunity existing among humans. Moreover, curative methods are still unknown. Persons suffering from cardiovascular diseases are at the highest risk of COVID-19. At present, there is no evidence for its transmission route through vaginal delivery or through breastfeeding, although newborn infants should be protected from exposure to infected subjects. ${ }^{5}$

\section{Structure of SARS-CoV-2 or Novel Coronavirus}

The SARS-CoV-2 is a spherical-shaped, positive, singlestranded ribonucleic acid (RNA) viral particle. This virus has spiked projections. It has been derived from Latin "corona" which means "crown." This conforms to their shape resembling a royal crown under an electron microscopic view. These are enveloped viral particles. The envelope is composed of a lipid bilayer, which is derived from the host cell membrane with structural proteins embedded within this envelope. These include spike (S), membrane (M), envelope $(\mathrm{E})$, and nucleocapsid $(\mathrm{N})$ proteins. The "N" protein is bound to the RNA forming nucleoprotein. $\mathrm{S}$ protein is a glycosylated protein forming homotrimeric spikes. This protein mediates the entry of viruses within host cells. The M protein is responsible for providing the virus its shape and is found in the largest amount. The E protein causes the release of viral particles from host cells. The CoV genome is the second largest among all RNA viruses. Its weight ranges between 26 to 32 kilobases (kb). ${ }^{1}$ Angiotensin-converting enzyme (ACE)-2 associated genes are responsible for various biological processes such as viral metabolism and immunological responses. Elevated ACE-2 levels have been found in oral epithelial cells and intrapulmonary airway but not among nonsmokers or those who had quit the habit of smoking. The binding process has been demonstrated between the SARS-CoV-2 spike (S) glycoprotein receptor-binding domain (RBD) and ACE-2 receptor. The " $\mathrm{"} \mathrm{protein} \mathrm{of}$ COVID-19 has structural similarity with SARS-CoV; hence, it exploits the ACE-2 receptor for infection among hosts. ${ }^{3}$

\section{Respiratory Diseases in COVID-19}

Analysis of viral genome analysis has suggested that SARSCoV-2 is a recombinant virus between bat and an unidentified origin coronavirus. The human transmission is thought to be possibly taken place from animals. However, there is still an inconclusiveness regarding the exact animal origins of human beings from bats, snakes, or any other animal in a chain of transmission. But there are positive findings about this virus from samples obtained from the seafood industry and markets. Respiratory droplets derived from coughing or sneezing are primary sources for human-to-human transmission. Most frequently reported symptoms of respiratory illnesses due to COVID-19 include higher fever than $38.1^{\circ} \mathrm{C}$ (98\% cases), severe cough (in 76\% cases), and severe fatigue or myalgia (observed in $44 \%$ patients). Dyspnea appears in 55\% infected subjects after 8 days and is the first severe complication of this disease. Also, diarrhea, headache, dyspnea, and hemoptysis have been reported as other clinical symptoms of COVID-19. ${ }^{2}$

The pneumonia caused by SARS-COV-2 is a highly contagious condition. Zhou et al conducted a study on 62 patients with COVID-19 pneumonia in Wuhan, China. They found that symptoms of fever (87.1\% cases), cough with sputum $(45.2 \%$ cases), pain in muscles (32.3\% cases), difficulty in breathing ( $24.2 \%$ cases), fatigue (22.6\% cases), and gastrointestinal symptoms such as pain in abdomen and diarrhea $(14.5 \%$ cases) constitute presenting symptoms of COVID-19 infection. These findings suggested the binding of viral particle to ACE receptor, which is highly expressed in the gastrointestinal tract of humans. These investigators found diverse patterns using computed tomography (CT) among COVID-19 pneumonia-affected patients. These include consolidation, reticular pattern, vacuolar sign, subpleural lines, and bronchial changes such as distortion, pleural retraction sign, and pleural effusion. Mixed patterns are found to affect both lung interstitium and parenchyma. ${ }^{6}$ Chest imaging shows bilateral ground-glass opacities. ${ }^{5}$

The Coronavirus pandemic has been preceded by two epidemics caused by betacoronavirus family, namely, MERSCoV and SARS-CoV-2. ${ }^{7}$

\section{Effects of Smoking and Alcohol in Disease Progression in SARS-CoV-2 Infection}

Smoking has been closely linked with adverse prognosis due to its bad effects on pulmonary health and the immune system. Such an association has been earlier seen in the MERS-CoV epidemic. However, there have been contradictory findings reported by few investigations in COVID-19. ${ }^{8}$ 
Table 1 Overview of studied literature

\begin{tabular}{|l|l|l|}
\hline Study (year) & Study design & $\begin{array}{l}\text { Smoking/alcohol habit-whether } \\
\text { significantly associated with disease severity }\end{array}$ \\
\hline${\text { Zhou et } \text { al }^{9}}$ & Retrospective study performed at multiple centers & $p=0.21$ \\
\hline Zhang et al $^{10}$ & Retrospective study, single center & $p=0.20$ \\
\hline Lippi and Henry $^{11}$ & Meta-analysis & $p=0.254$ \\
\hline Lai et al $^{5}$ & Retrospective, single center & Pneumonia reported in smokers \\
\hline Cohen $^{13}$ & Retrospective study & Alcohol shows low risk for respiratory illnesses \\
\hline
\end{tabular}

Due to these existing variations in published data, we performed a systematic analysis of studies involving the risk factors of smoking and alcohol consumption and their impact on the severity of COVID-19 infection among hospitalized subjects. A literature search was conducted on search engines PubMed and ScienceDirect, using the following terms for conducting a literature search: "AND" and "OR." Terms searched included "Smoking" AND "COVID-19"; "Smoking” OR “COVID-19"; "Alcohol” AND "COVID-19” and "Alcohol” OR “COVID-19," etc. All studies published in 2019 and 2020 were included in this systematic review. A total of five studies were included in this review analysis ( - Table $\mathbf{1}$ ).

Zhou et al found no statistically significant difference between the rate of smoking among those who survivors and nonsurvivors $(p$-value $=0.21) .{ }^{9}$ Similar findings were published by Zhang et al in 140 COVID-19 patients. They showed that of severe cases, smokers constituted $3.4 \%$, while $6.9 \%$ of cases were former smokers. In contrast to nonserious COVID-19 patients, there were no current smokers and 3.7\% were ones who quit smoking. ${ }^{10}$

Lippi and Henry in their meta-analysis observed that there is a contradiction regarding whether the smoking habit was associated with COVID-19 severity. Many investigators reported with no statistical association between active smoking and disease severity. A total of five studies were included in this meta-analysis, comprising 1,399 COVID-19 patients 288 (20.6\% cases) who suffered from severe disease. Only one study demonstrated that active smoking was a significant predictor of COVID-19 disease severity. However, the rest of the studies (total four in number) showed no statistically significant association. Although smokers are at higher risk, no significant association could be found between active smoking habit and SARS-CoV-2 severity on pooling data of studies included (odds ratio $[\mathrm{OR}]=1.69 ; 95 \%$ confidence interval $[\mathrm{CI}]=0.41-6.92$ and $p=0.254$ ). However, decreased ACE-2 levels have been reported in COVID-19 patients. ${ }^{11}$

One of the common measures employed by governments all over the world is isolation and distancing. However, longterm isolation can result in stress due to which chronic alcohol use increases. There is also a risk of relapse among past alcohol users and an increase in alcohol abuse. ${ }^{12}$

Consumption of alcohol concomitantly with smoking suppresses host immunity. This increases the risk of infections of the upper respiratory tract. There is evidence that the consumption of alcohol raises the chances of infection. Various complications associated with alcoholism include hepatic cirrhosis, deficiency of nutrients, and personal hygiene along with lifestyle changes. In a study conducted by Cohen, 36\% nonsmokers, $40 \%$ light smokers (consuming one to fifteen cigarettes per day), and $48 \%$ heavy smokers (consuming more than fifteen cigarettes per day) developed cold symptoms. This study concluded that smoking habit was associated with an increased risk of infection of the upper respiratory tract, while consumption of alcohol is associated with a reduced risk of respiratory illness. However, this correlation is completely dependent on dosages and their response. ${ }^{13}$

Patwardhan et al in their study concluded that the long isolation period, which is observed in COVID-19 spread, can create mental stress, leading to increase the frequency of smoking and even relapse in individuals who had quit the habit. ${ }^{14}$ Lai et al in their study observed that pneumonia was diagnosed in older patients who had higher smoking prevalence. They reported systemic conditions of hypertension (in $20.5 \%$ cases) and diabetes (in $14.4 \%$ cases). It was also observed that similar viral loads were found in both symptomatic and asymptomatic carriers. However, high-viral load was detected from patients with severe manifestations. ${ }^{5}$

Literature has proven studies to show that carcinogenesis is often associated with low-immunological status, which is characterized by overexpression of immunosuppressive cytokines along with suppressed induction of proinflammatory signals associated with apoptosis, impairment of maturation of dendritic cell, and increased immunosuppressive leukocytic populations. These chains of events are contradictory to presentations in severe COVID-19 patients. This difference in susceptibility along with prognosis is due to higher rates of smoking habits in patients with cancer. Also, tobacco usage has been found to significantly increase gene expression of ACE-2, which acts as a binding receptor for SARS-CoV-2. This can very well explain elevated susceptibility toward COVID-19 infection among smokers. Besides, smoking cigarettes is one of the leading reasons for COPD. COPD has been labeled as an independent risk factor in severe COVID-19 infections. ${ }^{15}$

Transmission of SARS-CoV-2 has been attributed via respiratory droplets and contamination of surfaces and hands. This microbial strain can persist upon inanimate surfaces for up to 9 days period at room temperature. As per recommendations by WHO, environmental cleaning and disinfection procedures should be followed constantly and correctly. Thorough cleaning of surrounding surfaces using water and detergents along with the application of common 
hospital-level disinfectants are both effective and sufficient for eradicating the virus. However, effective inactivation can be achieved by the use of surface disinfectants like $0.5 \%$ hydrogen peroxides, $0.1 \%$ sodium hypochlorite, or $62 \%$ to $71 \%$ ethanol. Carrier tests have shown that the use of $62 \%$ to $71 \%$ ethanol reduces viral load by 2.0 to $4.0 \log _{10 .}{ }^{12}$ As much as $0.1 \%$ to $0.5 \%$ sodium hypochlorite and $2 \%$ glutaraldehyde also demonstrates a decrease up to more than $3 \log _{10}$. All of the above-mentioned surface disinfectants show their effectiveness within 1-minute duration of application. Current testing methods employed for SARS-CoV-2 include polymerase chain reaction (PCR)-based assay. ${ }^{16}$

\section{Conclusion}

The SARS-CoV-2 or COVID-19 infection is an ongoing pandemic that is infecting increasingly numbers of people across the world. At present, there is no specific treatment, and it is expected to come after some time. Preventive and protective measures observed at individual levels lessen the risk of acquiring this disease. The virus-infected hosts have widely different metabolic systems, which are largely determined by dietary factors, nutritional level, age at time of infection, gender, underlying medical or systemic conditions, lifestyles and, most importantly, environmental factors. These variations govern the body's response to the clinical severity of COVID-19 disease infection. Thus, an individual's assessment of these factors is right now the best way in dealing with the SARS-CoV-2 pandemic. Studies utilizing data obtained from positive disease shows that active smoking can lead to adverse outcomes but was not significantly associated with a greater risk of progression toward COVID-19 severe disease. Further, due to the limited availability of data research should be performed to assess the outcome of smoking and consumption of alcohol on the outcome of COVID-19 severity.

Conflict of Interest

None declared.

\section{References}

1 Ashour HM, Elkhatib WF, Rahman MM, Elshabrawy HA. Insights into the recent 2019 novel coronavirus (SARS-CoV-2) in light of past human coronavirus outbreaks. Pathogens 2020;9(03): 186-191

2 Gasmi A, Noor S, Tippairote T, Dadar M, Menzel A, Bjorklund G. Individual risk management strategy and potential therapeutic options for the COVID-19 pandemic. Clin Immunol 2020 (e-pub ahead of print). Doi: 10.1016/j.clim.2020.108409

3 Emami A, Javanmardi F, Perbonyeh N, Akbari A. Prevalence of underlying disease in hospitalized patients with COVID-19: a systematic review and meta-analysis. Arch Acad Emergency Med 2020;8(01):235-249

4 Wang J, Luo Q, Chen R, Chen T, Li JX. Susceptibility analysis of COVID-19 in smokers based on ACE2. Preprints 2020 (e-pub ahead of print). Doi: 10.20944/preprints202003.0078.V1

5 Lai CC, Liu YH, Wang CY, et al. Asymptomatic carrier state, acute respiratory disease, and pneumonia due to severe acute respiratory syndrome coronavirus 2 (SARS-CoV-2): facts and myths. J Microbiol Immunol Infec 2020;53(03):404-412

6 Zhou S, Wang Y, Zhu T, Xia L. CT features of coronavirus disease 2019 (COVID-19) pneumonia in 62 patients in Wuhan, China. AJR Am J Roentgenol 2020;214(06):1287-1294

7 Zhao W, Zhong Z, Xie X, Yu Q, Liu J. Relation between chest CT findings and clinical considerations of coronavirus disease (COVID-19) pneumonia: A multicenter study. AJR 2020;4:1-6

8 Vardavas CI, Nikitara K. COVID-19 and smoking: a systematic review of the evidence. Tob Induc Dis 2020;18:20-24

9 Zhou F, Yu T, Du R, et al. Clinical course and risk factors for mortality of adult inpatients with COVID-19 in Wuhan, China: a retrospective cohort study. Lancet 2020;395(10229): 1054-1062

10 Zhang JJ, Dong X, Cao YY, et al. Clinical characteristics of 140 patients infected with SARS-CoV-2 in Wuhan, China. Allergy 2020;75(07):1730-1741

11 Lippi G, Henry BM. Active smoking is not associated with severity of coronavirus disease 2019 (COVID-19). Eur J Intern Med 2020; 75:107-108

12 Clay JM, Parker MO. Alcohol use and misuse during the COVID-19 pandemic: a potential public health crisis? Lancet Public Health 2020;5(05):e259

13 Cohen S, Tyrrell DAJ, Russell MAH, Jarvis MJ, Smith AP. Smoking, alcohol consumption, and susceptibility to the common cold. Am J Public Health 1993;83(09):1277-1283

14 Patwardhan P. COVID-19: risk of increase in smoking rates among England's 6 million smokers and relapse among England's 11 million ex-smokers. BJGP Open 2021;5(1):bjgpopen21X101161

15 Xia Y, Jin R, Zhao J, Li W, Shen H. Risk of COVID-19 for patients with cancer. Lancet Oncol 2020;21(04):e180

16 Kampf G, Todt D, Pfaender S, Steinmann E. Persistence of coronaviruses on inanimate surfaces and their inactivation with biocidal agents. J Hosp Infect 2020;104(03):246-251 